Technische

Universität

Berlin

\author{
Liudmila Tumash, Elena Panteley, Anna Zakharova, Eckehard \\ Schöll
}

\title{
Synchronization patterns in Stuart-Landau networks: a reduced system approach
}

Open Access via institutional repository of Technische Universität Berlin

Document type

Journal article | Accepted version

(i. e. final author-created version that incorporates referee comments and is the version accepted for publication; also known as: Author's Accepted Manuscript (AAM), Final Draft, Postprint)

This version is available at

https://doi.org/10.14279/depositonce-14983

Citation details

Tumash, L., Panteley, E., Zakharova, A., Schöll, E. Synchronization patterns in Stuart-Landau networks: a reduced system approach. Eur. Phys. J. B 92, 100 (2019). https://doi.org/10.1140/epjb/e2019-90483-5.

This is a post-peer-review, pre-copyedit version of an article published in The European Physical Journal B. The final authenticated version is available online at: http://dx.doi.org/10.1140/epjb/e2019-90483-5.

Terms of use

This work is protected by copyright and/or related rights. You are free to use this work in any way permitted by the copyright and related rights legislation that applies to your usage. For other uses, you must obtain permission from the rights-holder(s). 


\title{
Synchronization patterns in Stuart-Landau networks: a reduced system approach
}

\author{
Liudmila Tumash ${ }^{1, \text { a }}$, Elena Panteley ${ }^{2}$, Anna Zakharova ${ }^{1}$, and Eckehard Schöll ${ }^{1}$ \\ ${ }^{1}$ Institut für Theoretische Physik, Technische Universität Berlin, Hardenbergstraße 36, 10623 \\ Berlin, Germany \\ ${ }^{2}$ Laboratoire des signaux et systèmes, CNRS, 3 rue Joliot Curie, 91192 Gif-sur-Yvette, \\ France
}

\begin{abstract}
We study networks with coupled phase and amplitude dynamics. In particular, we investigate a ring of Stuart-Landau oscillators. For symmetry-conserving coupling we observe cluster synchronization. We show that the dimension of the dynamical system can be substantially reduced by projecting the system onto the subspace corresponding to the unstable eigenvalues of the linear part of the network dynamics.
\end{abstract}

\section{Introduction}

Originally being a branch of the graph theory, the study of networks triggered a burst of interest of the scientists from different disciplines after 1736. In this year the Swiss mathematician Leonhard Euler presented the solution to the Königsberg bridge problem. Nowadays, network systems are often encountered in both scientific and everyday life. They can represent objects on both Euclidian space, e.g., highways, subway systems, electric power grids, or they can refer to an abstract space, e.g., network of friendships, collaborations etc. We can also consider ourselves as entities of global social networks of different kinds. Although the nature of each system and the interconnections among its units differ drastically from one discipline to another, they are characterized by common features from the mathematical viewpoint.

Networks can be considered as sets of nodes, exhibiting some linear or nonlinear dynamic behavior. These nodes are connected via links, which represent interactions among them. The interplay of the individual dynamics and the network topology determines the large-scale collective behavior. Thereby, the individual nodes may reorganize relative to the emerging collective behaviour. This can be considered as a hierarchical process with the macroscopic level and the microscopic level, corresponding to the large-scale network behavior and the dynamics from the viewpoint of the constituting individual systems, respectively [1]. One of the first studies devoted to the synchronization behavior of coupled nonlinear oscillators goes back to 1934 [2]. The network behavior at the microscopic level is well-studied in dynamic control theory through the concept of synchronization [3-11].

\footnotetext{
${ }^{a}$ e-mail: liudmilatumash@gmx.de
} 
In the present study we analyse the emergent behavior for a ring of Stuart-Landau oscillators, which is a generic model for nonlinear oscillators near a Hopf bifurcation. We have chosen the nonlocal ring topology since it is widely studied in the context of synchronization patterns, and serves as a paradigm for nonlocal topologies, going beyond the simple case of local coupling. Moreover, many results can be obtained analytically for the chosen topology. We show that the dimension of the dynamical system can be substantially reduced, which facilitates numerical simulations, by projecting the system onto the subspace corresponding to the unstable eigenvalues of the linear part of the network dynamics. First, similar to $[1,12,13]$ we decompose the dynamic variables into a mean-field part corresponding to motion on the synchronization manifold $\mathbf{z}_{\mathbf{s}}$ and a synchronization error $\mathbf{e}$. The linear part of the network dynamics, after transforming to Jordan normal form, can be split into a low-dimensional synchronization manifold which is associated with the unstable directions of the fixed point in the origin from which the synchronized limit cycle oscillations have bifurcated, and hence with those eigenvalues which have positive real parts, and a transverse subspace of the synchronization error, which is associated with eigenvalues with negative real parts, leading to an asymptotically damped synchronization error.

This paper is organized as follows. First, we present the Stuart-Landau model. Then, we define the matrix which determines the stability of the linear part of the system and study its properties. Further, we decompose the system in two parts: synchronization error and emergent dynamics. We introduce new coordinates and derive the subsystem of reduced order for arbitrary coupling and for the special case of local coupling. Finally, we demonstrate numerical results for various system sizes, which verify that the lower-order system well preserves the properties of the original system. Here we deal with cluster states or standing-wave states, which asymptotically collapse towards complete synchrony. Additionally, we discuss the mechanisms providing this transition towards synchrony.

\section{Model}

In this article we study a nonlocally coupled ring of identical Stuart-Landau oscillators which represent the normal form of a nonlinear oscillator near a supercritical Hopf bifurcation. We consider a ring network of $N$ Stuart-Landau oscillators [14-24], $j \in$ $\{1, \ldots, N\}$, which are coupled with the strength $\sigma>0$ to their $P$ nearest neighbors in each direction $(r=P / N$ defines the dimensionless coupling range):

$$
\dot{z}_{j}=f\left(z_{j}\right)+\frac{\sigma}{2 P} \sum_{k=j-P}^{j+P}\left(z_{k}-z_{j}\right),
$$

where all indices are modulo $N$,

$$
f\left(z_{j}\right)=\left(\lambda+i \omega-\left|z_{j}\right|^{2}\right) z_{j}
$$

and $z_{j}=x_{j}+i y_{j}=r_{j} e^{i \phi_{j}} \in \mathbb{C}$, with $x_{j}, y_{j}, r_{j}, \phi_{j} \in \mathbb{R}$, and $\lambda, \omega>0$.

Without coupling (i.e., $\sigma=0$ ) the system undergoes a Hopf bifurcation at $\lambda=0$, so that for $\lambda>0$ a single Stuart-Landau oscillator performs self-sustained oscillations with frequency $\omega$ and follows the limit cycle trajectories with the radius $r_{j}=\sqrt{\lambda}$. In this case the unique fixed point $\left(x_{j}=y_{j}=0\right)$ is unstable and the periodic orbit $z_{j}(t)=r_{j} e^{i \omega t}$ is rotationally $\left(S^{1}\right)$ invariant.

If the coupling strength $\sigma$ is strong, then the oscillators globally in-phase synchronize [29], for weaker coupling collective behavior in the form of various synchronization patterns, for instance cluster synchronization [7], may emerge. Note that the 
coupling term in Eq. (1) conserves the $S^{1}$ symmetry, i.e., it does not break the rotational symmetry $\left(z_{j} \rightarrow z_{j} e^{i \psi}\right)$ of the system. Otherwise, for example if only the real parts $\operatorname{Re}\left(z_{k}\right)-\operatorname{Re}\left(z_{j}\right)$ were coupled, this would lead to the emergence of nontrivial inhomogeneous steady states $z_{j} \neq 0$, e.g., oscillation death [25-27].

In this paper our objective is to analyze the dynamics of such a Stuart-Landau network in the case when the coupling is not strong enough to ensure complete in-phase (isochronous) synchronization. In this case we observe more general synchronization patterns where the ring network of length $L$ supports a standing wave with wave vector $k=2 \pi / L$, i.e., the elements oscillate with different amplitudes. We develop a method to reduce the dimension of the dynamical system by projecting it onto the subspace corresponding to the unstable eigenvalues of the linear part of the network dynamics.

\section{Method}

With the $N$-dimensional complex column vector $\mathbf{z}=\operatorname{col}\left(z_{1}, \ldots z_{N}\right)$ we can rewrite the network of Stuart-Landau oscillators Eq. (1) in the vector form as

$$
\dot{\mathbf{z}}=-\mathbf{Z}^{(2)} \mathbf{z}+(\lambda+i \omega) \mathbf{z}-\frac{\sigma}{2 P} \mathbf{L} \mathbf{z},
$$

where $\mathbf{Z}^{(2)}=\operatorname{diag}\left(\left|z_{1}\right|^{2},\left|z_{2}\right|^{2}, \ldots,\left|z_{N}\right|^{2}\right)$ is an $N \times N$ diagonal matrix and the Laplacian matrix $\mathbf{L}$ contains all the terms due to coupling between the oscillators

$$
\mathbf{L}=\left[\begin{array}{ccccccc}
2 P & -1 & \cdots & 0 & \cdots & -1 & \cdots \\
-1 & 2 P & -1 & \cdots & 0 & \cdots & -1 \\
\cdot & . & . & \ddots & . & . & . \\
\cdot & . & . & . & \ddots & . & . \\
\cdot & \cdot & . & . & . & \ddots & . \\
-1 & \cdots & 0 & \cdots & -1 & \cdots & 2 P
\end{array}\right]
$$

The Laplacian matrix is defined as $\mathbf{L}=\mathbf{D}-\mathbf{A}$, where $\mathbf{A}$ and $\mathbf{D}$ are the adjacency and the degree matrix, respectively. In the case of ring networks with $P$ neighbours in each direction, the degree matrix has a simple diagonal form $\mathbf{D}=2 P \mathbb{I}_{\mathbf{N}}$ with the $N \times N$ unity matrix $\mathbb{I}_{\mathbf{N}}$, while $\mathbf{A}$ is a circulant matrix.

Next, we combine all linear terms that appear in Eq. (3) and define a matrix $\tilde{\mathbf{L}}=-\mathbf{L}+\frac{\lambda+i \omega}{\sigma} 2 P \mathbb{I}_{\mathbf{N}}$, hence Eq. (3) takes the form

$$
\dot{\mathbf{z}}=-\mathbf{Z}^{(2)} \mathbf{z}+\frac{\sigma}{2 P} \tilde{\mathbf{L}} \mathbf{z} .
$$

Synchronization properties of the network are closely connected with the properties of the matrix $\tilde{\mathbf{L}}$, in particular, the synchronous solution of the network Eq. (5) is stable if $N-1$ eigenvalues of this matrix are negative [28,29]. First we summarize the properties of the matrix $\tilde{\mathbf{L}}$ that we will use in the sequel.

\subsection{Properties of the matrix $\tilde{\mathrm{L}}$}

(i) Matrix $\tilde{\mathbf{L}}$ is similar to the adjacency matrix $\mathbf{A}$ :

$$
\tilde{\mathbf{L}}=\mathbf{A}+\left(-2 P+\frac{\lambda+i \omega}{\sigma} 2 P\right) \mathbb{I}_{\mathbf{N}} .
$$


and therefore one can write the eigenvalues of $\tilde{\mathbf{L}}$ as

$$
\tilde{\Lambda}_{j}=\Lambda_{j}-2 P+\frac{\lambda+i \omega}{\sigma} 2 P .
$$

where $\Lambda_{j}$ are the eigenvalues of $\mathbf{A}$. Moreover, the matrices $\tilde{\mathbf{L}}$ and $\mathbf{A}$ have the same eigenvectors $\mathbf{v}_{\mathbf{j}}(j=1, \ldots, N)$.

Eigenvectors of the circulant adjacency matrix $\mathbf{A}$ are $\mathbf{v}_{\mathbf{j}}=\frac{1}{\sqrt{N}}\left(1, \omega_{j}, \ldots, \omega_{j}^{N-1}\right)$, where $\omega_{j}=e^{2 \pi i \frac{j}{N}}$.

(ii) The eigenvalues of the matrix $\tilde{\mathbf{L}}$ can be found analytically using the similarity of $\tilde{\mathbf{L}}$ to the circulant adjacency matrix A, see Eq. (6), which has the eigenvalues [30]

$$
\Lambda_{j}=\frac{\sin \left(j \pi \frac{2 P+1}{N}\right)}{\sin \left(j \pi \frac{1}{N}\right)}-1, \quad j \in\{1, \ldots, N\} .
$$

Solving the eigenvalue problem of the circulant adjacency matrix $\mathbf{A}$, one can infer how to choose the parameters $P$ and $\sigma$ in order to obtain $2 l+1$ solutions $\tilde{\Lambda}_{j}$ with positive real parts, where $l \in \mathbb{N}$, using Eq. (7):

$$
\Lambda_{j}+2 P\left(\frac{\lambda}{\sigma}-1\right)>0 .
$$

For convenience we relabel the indices of our eigenvalues in a symmetric fashion, assuming even $N$ for technical simplicity: $j=\left\{-\frac{N}{2}, \ldots, \frac{N}{2}-1\right\}$. Eq. (9) can be used to show that for a given number $2 l+1$ of solutions with positive real parts there exist appropriate values of the parameters $P$ and $\sigma$.

In order to justify that there is always an odd number of solutions to the inequality (9), let us introduce a constant $C=2 P\left(1-\frac{\lambda}{\sigma}\right)$ and rewrite (9)

$$
\Lambda_{j}>C \Leftrightarrow \frac{\sin \left(j \pi \frac{2 P+1}{N}\right)}{\sin \left(j \pi \frac{1}{N}\right)}>\tilde{C}, \quad j \in\left\{-\frac{N}{2}, \ldots, \frac{N}{2}-1\right\}
$$

where $\tilde{C}=C+1$ is a constant.

Let us analyse this inequality in general. For this purpose we consider some function $\rho(x)=\frac{\sin k x}{\sin x}$, where $k$ is an integer, and $|x|<\pi$. This function is singular at $x=0$, but the limit for $x \rightarrow 0$ exists: $\frac{\sin (k x)}{\sin (x)} \rightarrow k$. The function $\rho(x)$ assumes its maximum at $x=0$, and it is a symmetric function. A solution to the inequality $\rho(x)>\tilde{C}$ exists only if the constant $\tilde{C}<k$. In this case $x^{*}=0$ will always be a solution. Moreover, if $x^{*} \neq 0$ is a solution, then $-x^{*}$ also satisfies the inequality $\rho(x)>\tilde{C}$ due to the symmetry of $\rho(x)$.

In our case $k=2 P+1$ and $x=\frac{j \pi}{N}$. Referring to the explanation above we can conclude that if any solution exists for the inequality (10), then $j=0$ is always a solution. Moreover, if $j^{*} \neq 0$ is a solution, then $-j^{*}$ is also a solution. Thus, the total number of solutions is an odd number.

We define the matrix of the $2 l+1$ eigenvalues with positive real parts $\tilde{\boldsymbol{\Lambda}}_{+}$, where for convenience we label the indices in a symmetric fashion: $j=\{-l, \ldots, l\}$ :

$$
\tilde{\boldsymbol{\Lambda}}_{+}=\left[\begin{array}{ccccc}
\tilde{\Lambda}_{-l} & 0 & 0 & \cdots & 0 \\
0 & \tilde{\Lambda}_{-l+1} & 0 & \cdots & 0 \\
\cdot & . & \ddots & . & . \\
. & . & . & \ddots & . \\
0 & 0 & 0 & \cdots & \tilde{\Lambda}_{l}
\end{array}\right],
$$


where $\operatorname{Re}\left(\tilde{\Lambda}_{-l}\right), \ldots, \operatorname{Re}\left(\tilde{\Lambda}_{l}\right)>0$. Analogously we define the matrix $\tilde{\Lambda}_{-}$of all eigenvalues with negative real parts of the matrix $\tilde{\mathbf{L}}$, and the overall matrix $\tilde{\boldsymbol{\Lambda}}=\operatorname{diag}\left(\tilde{\boldsymbol{\Lambda}}_{+}, \tilde{\boldsymbol{\Lambda}}_{-}\right)$. Let $\mathbf{V}=\operatorname{col}\left[\mathbf{v}_{\mathbf{1}}, \ldots, \mathbf{v}_{\mathbf{N}}\right]$ be the $N \times N$ unitary matrix whose columns are composed of the eigenvectors corresponding to the eigenvalues in matrix $\tilde{\mathbf{\Lambda}}$. Using the fact that matrix $\tilde{\mathbf{L}}$ is symmetric we can represent it as follows

$$
\tilde{\mathbf{L}}=\mathbf{V} \tilde{\mathbf{\Lambda}} \mathbf{V}^{*} \text {. }
$$

where $\mathbf{V}^{*}$ is the adjoint matrix.

\subsection{Decomposition of the system into two parts}

Similar to [12,13], here we present a decomposition of the system into a part which corresponds to the reduced system, and a part with asymptotically vanishing dynamics. The first part describes the synchronous solution and the second describes the dynamics of the synchronization errors.

We assume that the matrix $\tilde{\mathbf{L}}$ has $m=2 l+1$ eigenvalues with positive real parts and use the decomposition of the extended Laplacian matrix $\tilde{\mathbf{L}}$ given by Eq. (12), i.e., we introduce the coordinate transformation $\boldsymbol{\xi}=\mathbf{V}^{*} \mathbf{z}$.

In these new coordinates the network dynamics can be written as

$$
\dot{\boldsymbol{\xi}}=\mathbf{V}^{*} \dot{\mathbf{z}}=-\mathbf{V}^{*} \mathbf{Z}^{(2)} \mathbf{z}+\frac{\sigma}{2 P} \mathbf{V}^{*} \tilde{\mathbf{L}} \mathbf{z}=-\mathbf{V}^{*} \mathbf{Z}^{(2)} \mathbf{V} \boldsymbol{\xi}+\frac{\sigma}{2 P} \tilde{\mathbf{\Lambda}} \boldsymbol{\xi}
$$

where we have used Eq. (12) and $\mathbf{z}=\mathbf{V} \boldsymbol{\xi}$. Note that the nonlinear part of Eq. (13) still contains the term $\mathbf{Z}^{(2)}$ and therefore depends on $\mathbf{z}$.

Similar to the decomposition of the matrix $\tilde{\boldsymbol{\Lambda}}$ we can decompose the matrix $\mathbf{V}$ into two parts as $\mathbf{V}=\left[\mathbf{V}_{+}, \mathbf{V}_{-}\right]$where the $N \times m$ and $N \times(N-m)$ matrices $\mathbf{V}_{+}$and $\mathbf{V}_{-}$, respectively, are constructed from eigenvectors corresponding to positive and negative eigenvalues, respectively. Similarly the $N$ dimensional vector $\boldsymbol{\xi}$ can be split into an $m$-dimensional vector $\boldsymbol{\xi}_{+}=\mathbf{V}_{+}^{*} \mathbf{z}$ and an $(N-m)$-dimensional vector $\boldsymbol{\xi}_{-}=\mathbf{V}_{-}^{*} \mathbf{z}$. Thus the network dynamics is split into two subsystems

$$
\begin{aligned}
& \dot{\boldsymbol{\xi}}_{+}=-\mathbf{V}_{+}^{*} \mathbf{Z}^{(2)} \mathbf{V} \boldsymbol{\xi}+\frac{\sigma}{2 P} \tilde{\boldsymbol{\Lambda}}_{+} \boldsymbol{\xi}_{+} \\
& \dot{\boldsymbol{\xi}}_{-}=-\mathbf{V}_{-}^{*} \mathbf{Z}^{(2)} \mathbf{V} \boldsymbol{\xi}+\frac{\sigma}{2 P} \tilde{\boldsymbol{\Lambda}}_{-} \boldsymbol{\xi}_{-} .
\end{aligned}
$$

It should be stressed that the dynamical properties of these two subsystems are essentially different since the matrix $\tilde{\Lambda}_{+}$is a diagonal positive matrix, while $\tilde{\Lambda}_{-}$is negative. The eigenvalues with positive real parts of the linear part $\tilde{\mathbf{L}}$ of Eq. (5) are precisely the unstable eigenvalues following from the

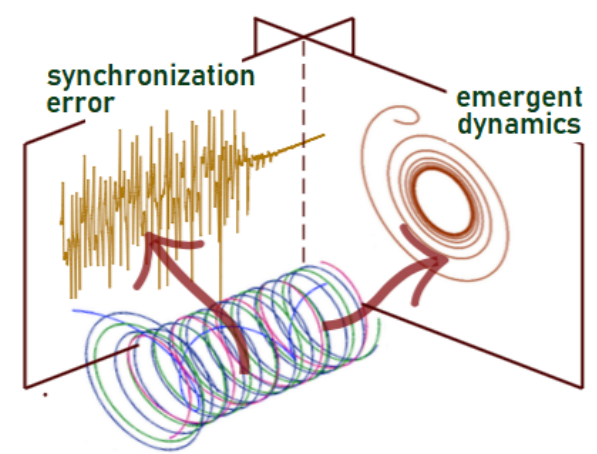

Fig. 1. Schematic illustration of the projection of the phase space trajectories onto the submanifold of emergent synchronous dynamics and the transverse subspace of synchronization error, which is schematically represented by its time series (horizontally: time, vertically: synchronization error). (C)[2017] IEEE. Reprinted, with permission, from E. Panteley and A. Loria [1]. 
linear stability analysis of the fixed point in the origin, hence the corresponding eigenvectors indicate the unstable directions of this fixed point, from which the synchronized limit cycle oscillations have bifurcated. They span the submanifold of the full phase space $\mathbb{C}^{N}$ in which the limit cycle oscillations are located.

Let us introduce the synchronization error coordinates $\mathbf{e}=\mathbf{V}_{-} \boldsymbol{\xi}_{-} \in \mathbb{C}^{N}$, and note that due to the properties of the unitary matrix $\mathbf{V}$ we can decompose the $N$ dimensional unit matrix as $\mathbb{I}_{\mathbf{N}}=\mathbf{V}_{-} \mathbf{V}_{-}^{*}+\mathbf{V}_{+} \mathbf{V}_{+}^{*}$, and therefore we can write $\mathbf{e}=$ $\mathbf{V}_{-} \boldsymbol{\xi}_{-}=\mathbf{V}_{-} \mathbf{V}_{-}^{*} \mathbf{z}=\left(\mathbb{I}_{\mathbf{N}}-\mathbf{V}_{+} \mathbf{V}_{+}^{*}\right) \mathbf{z}=\mathbf{z}-\mathbf{V}_{+} \boldsymbol{\xi}_{+}$, where we have used the definitions $\boldsymbol{\xi}_{-}=\mathbf{V}_{-}^{*} \mathbf{z}$ and $\boldsymbol{\xi}_{+}=\mathbf{V}_{+}^{*} \mathbf{z}$. Then, using the quadratic Lyapunov function $V(\mathbf{e})=\frac{1}{2} \mathbf{e}^{*} \mathbf{e}$ we can deduce that the synchronization error $\mathbf{e}(t) \rightarrow 0$ for $t \rightarrow \infty$ and hence $\boldsymbol{\xi}_{-}(t)$ asymptotically tends to zero as well (see Appendix).

Thus the subspace $\boldsymbol{\xi}_{+}$captures the generalized synchronous solutions, while the subspace $\boldsymbol{\xi}_{-}$describes the synchronization error which asymptotically for $t \rightarrow \infty$ vanishes, as schematically represented in Fig. 1 . This means that asymptotically $\mathbf{z}(t)$ is determined by $\boldsymbol{\xi}_{+}$, i.e., $\mathbf{z}(t) \approx \mathbf{V}_{+} \boldsymbol{\xi}_{+}(t)$. Hence we can asymptotically reduce the model Eq. (15) to the phase space spanned by $\boldsymbol{\xi}_{+}$.

To derive the equations of the reduced system in the case of vanishing synchronization error $\boldsymbol{\xi}_{-}=0$, we will now focus on the nonlinear part of the system Eq. (13):

$$
\begin{aligned}
\mathbf{V}_{+}^{*} \mathbf{Z}^{(2)} \mathbf{V} \boldsymbol{\xi} & =\mathbf{V}_{+}^{*} \mathbf{Z}^{(2)}\left[\mathbf{V}_{+}, \mathbf{V}_{-}\right]\left[\begin{array}{l}
\boldsymbol{\xi}_{+} \\
\boldsymbol{\xi}_{-}
\end{array}\right]=\mathbf{V}_{+}^{*} \mathbf{Z}^{(2)} \mathbf{V}_{+} \boldsymbol{\xi}_{+}=\sum_{i=-l}^{l} \mathbf{V}_{+}^{*} \mathbf{Z}^{(2)} \mathbf{V}_{\mathbf{k}_{\mathbf{i}}} \xi_{i} \\
& =\sum_{i=-l}^{l} \xi_{i} \mathbf{V}_{+}^{*} \mathbf{Z}^{*} \mathbf{V}_{\mathbf{k}_{\mathbf{i}}} \mathbf{z}=\sum_{i=-l}^{l} \xi_{i} \mathbf{V}_{+}^{*} \mathbf{Z}^{*} \mathbf{V}_{\mathbf{k}_{\mathbf{i}}} \mathbf{V} \boldsymbol{\xi}=\sum_{i=-l}^{l} \xi_{i} \mathbf{V}_{+}^{*} \mathbf{Z}^{*} \mathbf{V}_{\mathbf{k}_{\mathbf{i}}} \mathbf{V}_{+} \boldsymbol{\xi}_{+},
\end{aligned}
$$

where $\mathbf{v}_{\mathbf{k}_{\mathbf{i}}}$ is the $k_{i}$-th $N$-dimensional eigenvector from the unstable manifold and $\mathbf{V}_{\mathbf{k}_{\mathbf{i}}}=\operatorname{diag}\left(\mathbf{v}_{\mathbf{k}_{\mathbf{i}}}\right)$ is a $N \times N$ diagonal matrix obtained by placing the elements of the vector $\mathbf{v}_{\mathbf{k}_{\mathbf{i}}}$ along the diagonal. Indices $k$ and $i$ depend on the coupling parameter $P$ and solution number $i$, respectively. We have also used the property $\mathbf{Z}_{\mathbf{k}_{\mathbf{i}}}=\mathbf{V}_{\mathbf{k}_{\mathbf{i}}} \mathbf{z}$.

We focus now on the $j$-th component of vector $\boldsymbol{\xi}_{+}$denoted as $\xi_{j, n l}^{+}$:

$$
\begin{aligned}
\dot{\xi}_{j, n l}^{+}=-\sum_{i=-l}^{l} \xi_{i} \mathbf{V}_{\mathbf{k}_{\mathbf{j}}}^{*} \mathbf{Z}^{*} \mathbf{V}_{\mathbf{k}_{\mathbf{i}}} \mathbf{V}_{+} \boldsymbol{\xi}_{+} & =-\sum_{i=-l}^{l} \xi_{i} \mathbf{z}^{*} \mathbf{V}_{\mathbf{k}_{\mathbf{j}}}^{*} \mathbf{V}_{\mathbf{k}_{\mathbf{i}}} \mathbf{V}_{+} \boldsymbol{\xi}_{+} \\
& =-\sum_{i=-l}^{l} \xi_{i} \boldsymbol{\xi}_{+}^{*} \mathbf{V}_{+}^{*} \mathbf{V}_{\mathbf{k}_{\mathbf{j}}}^{*} \mathbf{V}_{\mathbf{k}_{\mathbf{i}}} \mathbf{V}_{+} \boldsymbol{\xi}_{+}=-\sum_{i=-l}^{l} \xi_{i} \boldsymbol{\xi}_{+}^{*} \mathbf{A}^{[\mathbf{j i}]} \boldsymbol{\xi}_{+},
\end{aligned}
$$

where $\mathbf{A}^{[\mathbf{j i}]}=\mathbf{V}_{+}^{*} \mathbf{V}_{\mathbf{k}_{\mathbf{j}}}^{*} \mathbf{V}_{\mathbf{k}_{\mathbf{i}}} \mathbf{V}_{+}$is an $m \times m$ matrix. With

$$
\mathbf{V}_{\mathbf{k}_{\mathbf{i}}} \mathbf{V}_{+}=\mathbf{V}_{\mathbf{k}_{\mathbf{i}}}\left(\mathbf{v}_{-\mathbf{k}_{\mathbf{l}}} \ldots \mathbf{v}_{\mathbf{0}} \ldots \mathbf{v}_{\mathbf{k}_{\mathbf{l}}}\right)=\frac{1}{\sqrt{N}}\left(\mathbf{v}_{\mathbf{k}_{\mathbf{i}}-\mathbf{k}_{\mathbf{1}}} \ldots \mathbf{v}_{\mathbf{k}_{\mathbf{i}}} \ldots \mathbf{v}_{\mathbf{k}_{\mathbf{i}}+\mathbf{k}_{\mathbf{l}}}\right)
$$

it follows that

$$
\mathbf{A}^{[\mathbf{j i}]}=\mathbf{V}_{+}^{*} \mathbf{V}_{\mathbf{k}_{\mathbf{j}}}^{*} \mathbf{V}_{\mathbf{k}_{\mathbf{i}}} \mathbf{V}_{+}=\frac{1}{N}\left(\begin{array}{c}
\mathbf{v}_{\mathbf{k}_{\mathbf{j}}-\mathbf{k}_{\mathbf{1}}}^{*} \\
\vdots \\
\mathbf{v}_{\mathbf{k}_{\mathbf{j}}}^{*} \\
\vdots \\
\mathbf{v}_{\mathbf{k}_{\mathbf{j}}+\mathbf{k}_{\mathbf{l}}}^{*}
\end{array}\right)\left(\mathbf{v}_{\mathbf{k}_{\mathbf{i}}-\mathbf{k}_{\mathbf{l}}} \ldots \mathbf{v}_{\mathbf{k}_{\mathbf{i}}} \ldots \mathbf{v}_{\mathbf{k}_{\mathbf{i}}+\mathbf{k}_{\mathbf{l}}}\right)
$$


The scalar product of two eigenvectors $\mathbf{v}_{\mathbf{a}}$ and $\mathbf{v}_{\mathbf{b}}$ is defined as:

$$
\mathbf{v}_{\mathbf{a}}^{*} \mathbf{v}_{\mathbf{b}}=\sum_{i=1}^{N} v_{(i, b-a)}= \begin{cases}1 & \text { if } b-a=0 \\ 0 & \text { if } b-a \neq 0\end{cases}
$$

Hence we can write the matrix $\mathbf{A}^{[\mathbf{j i}] \text { : }}$

$$
\mathbf{A}^{[\mathbf{j i}]}=\frac{1}{N}[\Psi(j, i, p, q)]_{p, q \in[-l, l]}, \quad \text { where } \Psi(j, i, p, q)=\left\{\begin{array}{ll}
1 & \text { if } k_{i}-k_{j}+k_{q}-k_{p}=0 \\
0 & \text { if } k_{i}-k_{j}+k_{q}-k_{p} \neq 0
\end{array},\right.
$$

here $p$ numbers the row, $q$ numbers the column (both numbered from $-l$ to $l$ ), $\Psi(j, i, p, q)$ is a binary variable. It follows that $[\Psi(j, i, p, q)]_{p, q \in[-l, l]}$ for fixed $p, q$ is an $m \times m$ diagonal matrix with entries 0 or $\frac{1}{N}$.

As a result one obtains the nonlinear part of the equations for the reduced system with an arbitrary coupling range $P$ :

$$
\dot{\xi}_{j, n l}=-\frac{1}{N} \sum_{i=-l}^{l} \sum_{p=-l}^{l} \sum_{q=-l}^{l} \xi_{i} \xi_{p}^{*} \xi_{q} \Psi(j, i, p, q)
$$

In order to complete the derivation of the reduced system, we consider the $j$-th component of the linear part of the Stuart-Landau model denoted as $\xi_{j, l i n}^{+}$(see Eq. 13)

$$
\dot{\xi}_{j, l i n}^{+}=\frac{\sigma}{2 P} \tilde{\Lambda}_{j}^{+} \xi_{j}
$$

In the following we skip the superscript + with respect to the variable $\xi_{j}$ for simplicity.

Combining the results obtained from Eq. (22) and Eq. (23), we obtain the full reduced system of equations:

$$
\dot{\xi}_{j}=-\frac{1}{N} \sum_{i=-l}^{l} \sum_{p=-l}^{l} \sum_{q=-l}^{l}\left[\xi_{i} \xi_{p}^{*} \xi_{q} \Psi(j, i, p, q)\right]+\frac{\sigma}{2 P} \tilde{\Lambda}_{j}^{+} \xi_{j},
$$

where $\tilde{\Lambda}_{j}^{+}$is the $j$-th diagonal element of matrix $\tilde{\Lambda}_{+}$.

\subsection{Special case: $P=1$}

In the case of local coupling $(P=1)$ we have $k_{l} \equiv l$. Thus the definition of $\Psi$ reduces to

$$
\Psi(j, i, p, q)= \begin{cases}1 & \text { if } q-p=j-i \\ 0 & \text { if } q-p \neq j-i\end{cases}
$$

This means that unity is placed on the $k$-th diagonal of matrix $\mathbf{A}^{[\mathbf{j i}]}$ with $k=j-i$. Then

$$
\boldsymbol{\xi}_{+}^{*} \mathbf{A}^{[\mathbf{j i}]} \boldsymbol{\xi}_{+}=\frac{1}{N} \sum_{k=\max (-l,-l+i-j)}^{\min (l, l+i-j)} \xi_{k}^{*} \xi_{k+j-i}
$$

As a result one obtains the reduced system of equations for $P=1$ :

$$
\dot{\xi}_{j}=-\frac{1}{N} \sum_{i=-l}^{l} \sum_{k=\max (-l,-l+i-j)}^{\min (l, l+i-j)}\left[\xi_{i} \xi_{k}^{*} \xi_{k+j-i}\right]+\frac{\sigma}{2 P} \tilde{\Lambda}_{j}^{+} \xi_{j}
$$




\section{Numerical results}

\subsection{Results for $N=12$}

In this section we illustrate the above results by a Stuart-Landau network of $N=12$ oscillators, which are locally coupled $(P=1)$ with the coupling strength $\sigma=5$. We fix the bifurcation parameter $\lambda=1$ and the oscillation frequency $\omega=2$. This is the smallest possible network for which we have observed standing waves. In Fig. 2 we show the space-time plots (top panel) and snapshots for $t=0$ (middle panel) and $t=T / 2$ (bottom panel), where $T=2 \pi / \omega$ is the period of oscillation, for two different network sizes, (a) $N=12$ (left column) and (b) $N=100$ (right column). In the snapshot for $N=12$ one can observe the standing-wave profile corresponding to a wavevector $k=2 \pi / L$ where $L$ is the length of the ring, such that nodes $\left\{1, \ldots, \frac{N}{2}\right\}$ and $\left\{\frac{N}{2}+1, \ldots, N\right\}$ are in anti-phase. Additionally, nodes $j$ and $\frac{N}{2}+1-j$ oscillate with the same phase and the same amplitude. These two symmetry features are a result of the specially prepared initial conditions, $x_{j}=1, y_{j}=-1$ for $j \in\left\{1, \ldots, \frac{N}{2}\right\}$ and $x_{j}=-1, y_{j}=1$ for $j \in\left\{\frac{N}{2}+1, \ldots, N\right\}$. Hence we expect that the number of clusters in such a network is determined by $\frac{N}{4}$. The comparison with a network of $N=100$ nodes (Fig.2 b) reveals that the standing-wave profile and the symmetry properties are retained.

This particular set of parameters results in $m=2 l+1=3$ eigenvalues with positive real parts in accordance with Eq. (9), hence

$$
\tilde{\boldsymbol{\Lambda}}_{+}=\left[\begin{array}{ccc}
0.1321+0.8 \mathrm{i} & 0 & 0 \\
0 & 0.4+0.8 \mathrm{i} & 0 \\
0 & 0 & 0.1321+0.8 \mathrm{i}
\end{array}\right]
$$

Since the coupling is local $(\mathrm{P}=1)$, we can apply Eq. (27) for the reduced system

$$
\left\{\begin{array}{c}
\dot{\xi}_{-1}=-\frac{1}{12}\left[\xi_{-1}\left(\left|\xi_{-1}\right|^{2}+2\left|\xi_{0}\right|^{2}+2\left|\xi_{1}\right|^{2}\right)+\xi_{0}^{2} \xi_{-1}^{*}\right]+(0.33025+2.0 \mathrm{i}) \xi_{-1} \\
\dot{\xi}_{0}=-\frac{1}{12}\left[\xi_{0}\left(2\left|\xi_{-1}\right|^{2}+\left|\xi_{0}\right|^{2}+2\left|\xi_{1}\right|^{2}\right)+2 \xi_{0}^{*} \xi_{1} \xi_{-1}\right]+(1.0+2.0 \mathrm{i}) \xi_{0} \\
\dot{\xi}_{1}=-\frac{1}{12}\left[\xi_{1}\left(2\left|\xi_{-1}\right|^{2}+2\left|\xi_{0}\right|^{2}+\left|\xi_{1}\right|^{2}\right)+\xi_{0}^{2} \xi_{1}^{*}\right]+(0.33025+2.0 \mathrm{i}) \xi_{1}
\end{array}\right.
$$

This system of equations describes the dynamics in the $(m=3)$-dimensional phase space of the reduced system. The results are depicted in Fig. 3 for the original system of $N=12$ (a), the reduced system of $m=2 l+1$ elements (b) and the lifted trajectories after back transformation to the $N=12$-dimensional phase space, using the relation $\mathbf{z}=\mathbf{V}_{+} \boldsymbol{\xi}_{+}$(c). As expected, we obtain for the original system $\frac{N}{4}=3$ limit cycles of different radii around the origin, corresponding to three clusters each of which consists of 4 nodes, two in-phase and two anti-phase. This is an unstable state, and hence, even if we start exactly on this invariant state, due to numerical rounding errors it disappears after some time and transforms into a completely in-phase synchronized state, a limit cycle of radius $r=\sqrt{\lambda}=1$. The reduced system is dominated by the variables which correspond to the eigenvalues with positive real parts $\tilde{\Lambda}_{-1}$ and $\tilde{\Lambda}_{1}$. They are equal but their corresponding eigenvectors $\mathbf{v}_{\mathbf{1}}$ and $\mathbf{v}_{-\mathbf{1}}$ form a complex conjugate pair. The variable $\xi_{0}$ tends to the stationary state $\xi_{0}=0$ and does not influence the dynamics of the reduced order model. After lifting the reduced system to the full system of dimension $N=12$ by applying the back transformation we obtain almost the same oscillatory behavior as in the original system. 

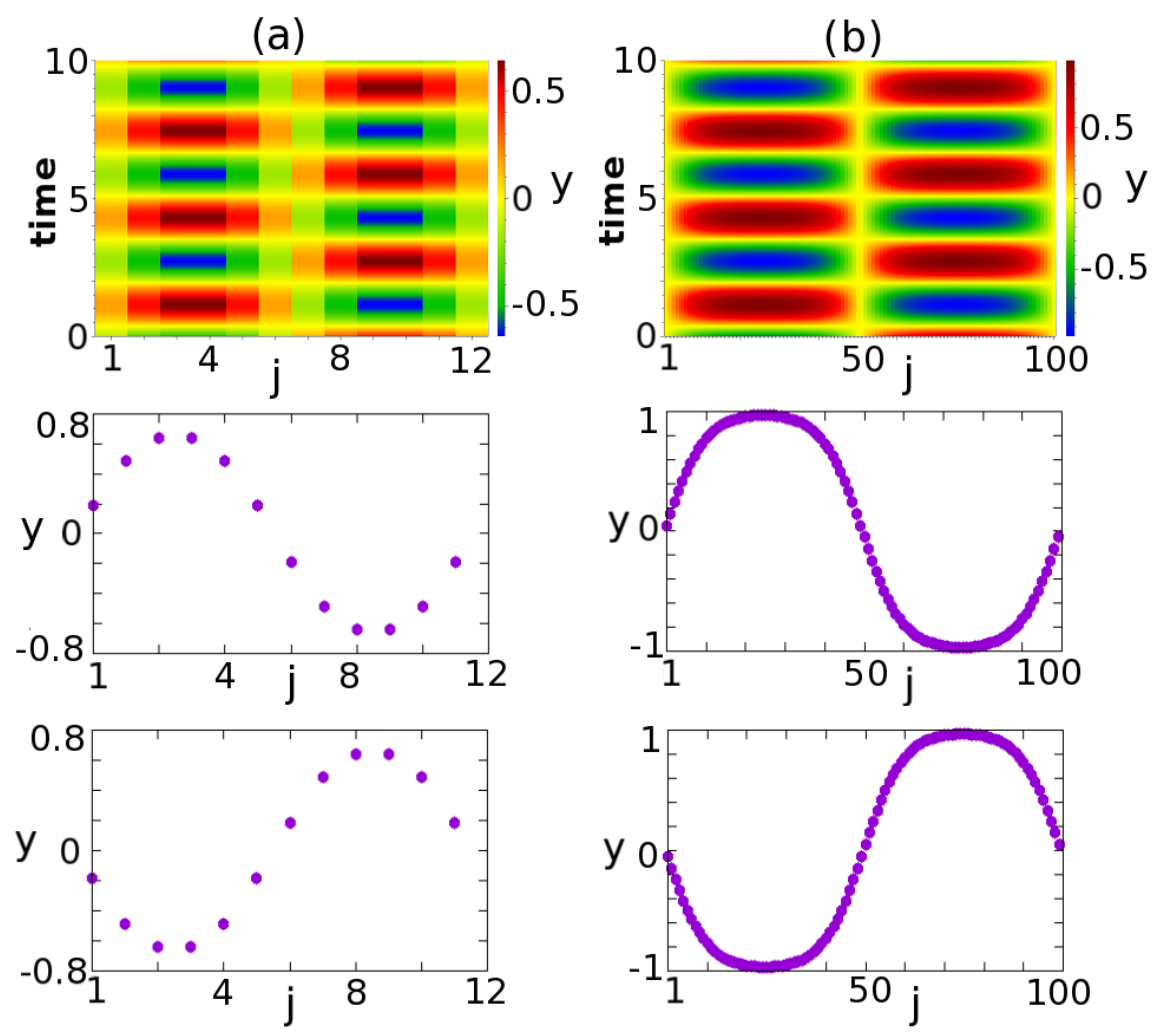

Fig. 2. Standing wave synchronization pattern of Stuart-Landau network: (a) $N=12$, $\sigma=5, P=1$, (b) $N=100, \sigma=14, P=4$. The top panels show space-time plots where $y=\operatorname{Im} z$ is color-coded, the middle and bottom panels show snapshots of $y$ at $t=0$ and $t=\frac{T}{2}$, respectively, where $T=2 \pi / \omega=\pi$. Other parameters: $\lambda=1, \omega=2$. Initial transients of $t=2 T$ have been skipped.

(a)

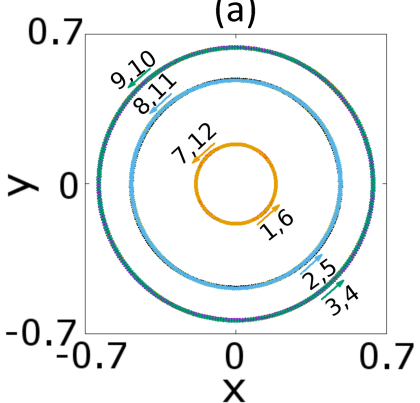

(b)

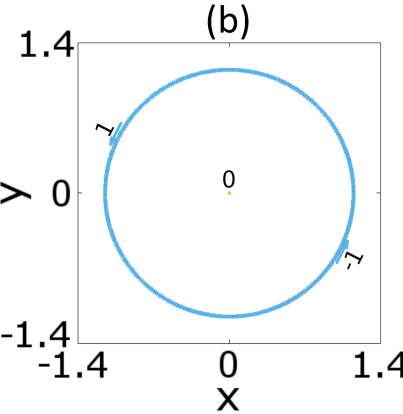

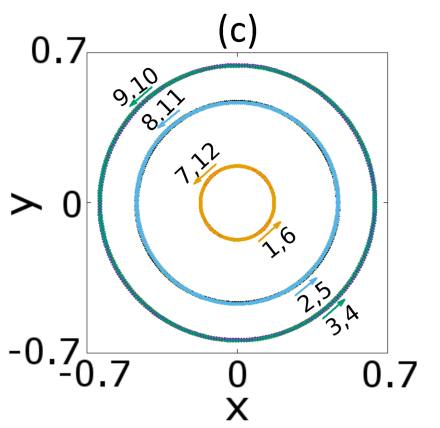

Fig. 3. Standing wave trajectories for $10 T \leqslant t \leqslant 13 T$ in the phase space $(x, y)$ of (a) original system of dimension $N=12$; (b) reduced system of $m=3$; (c) reduced system lifted to $N=12$. Other parameters: $\sigma=5, P=1, \lambda=1, \omega=2$. The limit cycles are labelled by the corresponding node indices.

After some time, due to numerical rounding errors, the system leaves the unstable standing wave pattern and asymptotically reaches a completely in-phase synchronized state, a limit cycle of radius $r=1$ (Fig. 4(a)). In panel (b) in the reduced system 
(a)

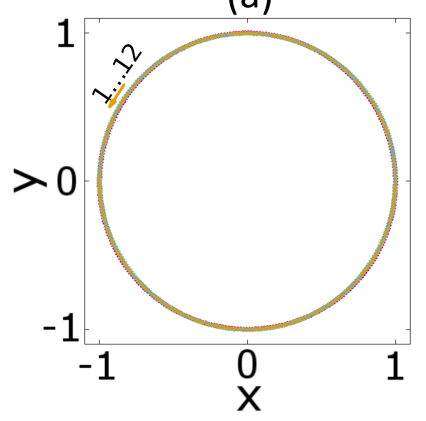

(b)

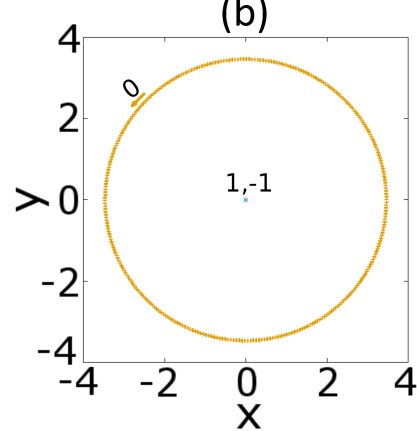

(c)

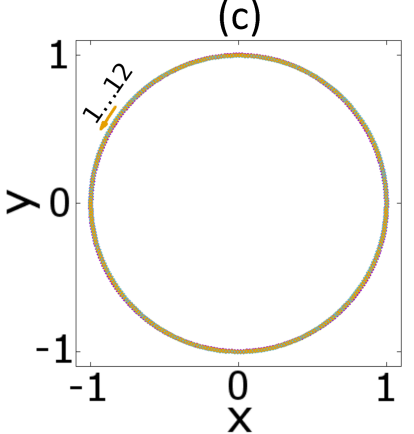

Fig. 4. Asymptotic fully synchronized trajectories for $30 T \leqslant t \leqslant 33 T$ in the phase space $(x, y)$ of (a) original system of $N=12$; (b) reduced system of $m=3$; (c) reduced system lifted to $N=12$. Other parameters: $\sigma=5, P=1, \lambda=1, \omega=2$.

we observe a limit cycle and a stationary point at the origin; here the limit cycle originates from $\xi_{0}$, and both variables $\xi_{1}$ and $\xi_{-1}$ collapse into the stationary state. The explanation of these differences for the reduced system in case of transient and asymptotic states follows in the next subsection. By lifting the reduced system into the full $N=12$ space, the original trajectory is recovered (Fig. 4(c)).

\subsection{Special cases of the reduced system}

In the case of full synchronization there is only one nonzero variable in the reduced system, which is $\xi_{0}$. Namely, if the original system is fully synchronized, then $z_{i}=\bar{z}$ $\forall i$. It follows that

$$
\begin{aligned}
& \xi_{j}=\mathbf{v}_{\mathbf{j}}^{*} \mathbf{z}=\frac{1}{\sqrt{N}} \sum_{i=0}^{N-1} \omega^{-i j} z_{i+1}=\frac{1}{\sqrt{N}} \bar{z} \sum_{i=0}^{N-1} \omega^{-i j}=0, \quad j \neq 0 \\
& \xi_{0}=\mathbf{v}_{\mathbf{0}}{ }^{*} \mathbf{z}=\frac{1}{\sqrt{N}} \sum_{i=0}^{N-1} z_{i+1}=\frac{1}{\sqrt{N}} \sum_{i=0}^{N-1} \bar{z}=\sqrt{N} \bar{z}
\end{aligned}
$$

Here $\omega \equiv \exp \left(2 \pi i \frac{1}{N}\right)$. This explains why the amplitude of $\xi_{0}$ for the completely synchronized limit cycle in the reduced system equals $\sqrt{N}$, since the synchronous solution $\bar{z}$ has amplitude $r=1$.

In the case of a standing wave with wavevector $k=2 \pi / L$, from the symmetry properties it follows that $z_{i}=-z_{i+N / 2}$. Hence

$$
\xi_{0}=\mathbf{v}_{\mathbf{0}}{ }^{*} \mathbf{z}=\frac{1}{\sqrt{N}} \sum_{i=1}^{N} z_{i}=\frac{1}{\sqrt{N}} \sum_{i=1}^{N / 2}\left[z_{i}+z_{i+N / 2}\right]=0
$$

In Fig. 5 we show the norm of the reduced variables $\left\|\xi_{0}\right\|,\left\|\xi_{1}\right\|$ and $\left\|\xi_{-1}\right\|$ vs. time, in order to detect the lifetime of the unstable standing wave state. It reveals an abrupt change of the reduced system dynamics at $t \approx 70(t \approx 22 T)$. Here the system attains complete synchronization, and thus the lifetime of the cluster state ends. It is also remarkable that its lifetime strongly depends on the system parameters and initial conditions. Of course, this abrupt change also shows up in the space-time plot. 
(a)

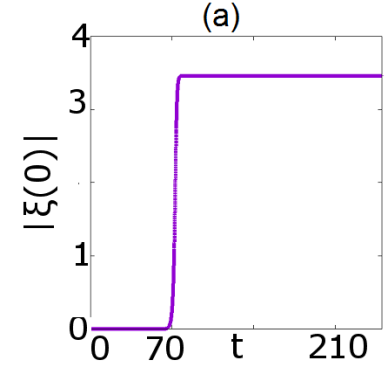

(b)

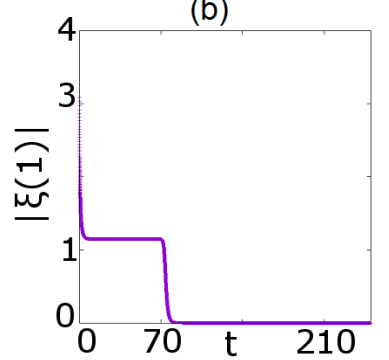

(c)

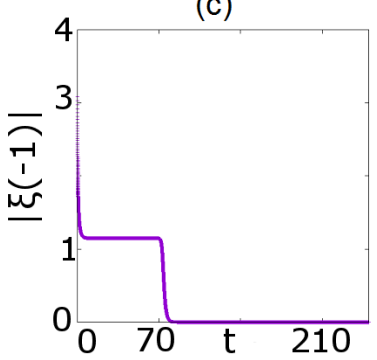

Fig. 5. Norm of the reduced system coordinates for $m=3$ vs time $t$ : (a) coordinate $\xi_{0}$ related to $\tilde{\Lambda}_{0} ;(\mathrm{b})$ coordinate $\xi_{1}$ related to $\tilde{\Lambda}_{1} ; \mathrm{c}$ ) coordinate $\xi_{-1}$ related to $\tilde{\Lambda}_{-1}$. Other parameters: $\sigma=5, P=1, \lambda=1, \omega=2$.

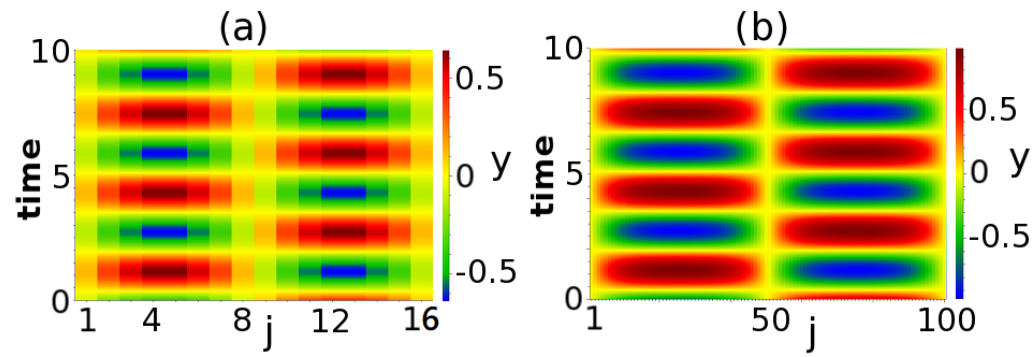

Fig. 6. Space-time plot for $2 T \leqslant t \leqslant 5 T$ for: (a) $N=16, \sigma=9, P=1$; (b) $N=100$, $\sigma=14, P=4$. Other parameters: $\lambda=1, \omega=2$. Initial transients of $t=2 T$ have been skipped.

\subsection{Results for $N=16$}

Next, we have applied our reduction method to a system of different size, $N=16$, and $\sigma=9$. All other parameters and the initial conditions remain the same. The space-time plot in Fig. 6 shows two antisymmetric domains forming a standing wave profile with $\frac{N}{4}=4$ limit cycles of different radii. The system is also periodic.

We perform the same reduction for the transient standing wave of $N=16$. In Fig. 7 one can see (a) the original system with 4 limit cycles formed by $N=16$ elements, (b) the reduced system which consists of a limit cycle formed by $\xi_{1}, \xi_{-1}$ and a stationary state of $\xi_{0}$, (c) lifted system obtained from the back transformation of the reduced system. Also here the lifted system perfectly reproduces the original system dynamics.

We have carried out a similar analysis for the completely in-phase synchronized asymptotic state of $N=16$, and also for transient and asymptotic states of networks with $N=20, N=24$, and $N=28$ elements. Here we observe that a larger system size leads to longer lifetimes of the transient standing-wave states.

\section{Conclusion}

We have considered a network of Stuart-Landau oscillators with $S^{1}$ symmetry-conserving coupling. Within a reduced system approach we showed that it is possible to reduce the dimension of the system, while preserving the collective dynamics. Our approach consists in projecting the network dynamics upon the synchronization manifold spanned by the eigenvectors corresponding to the unstable eigenvalues of the 

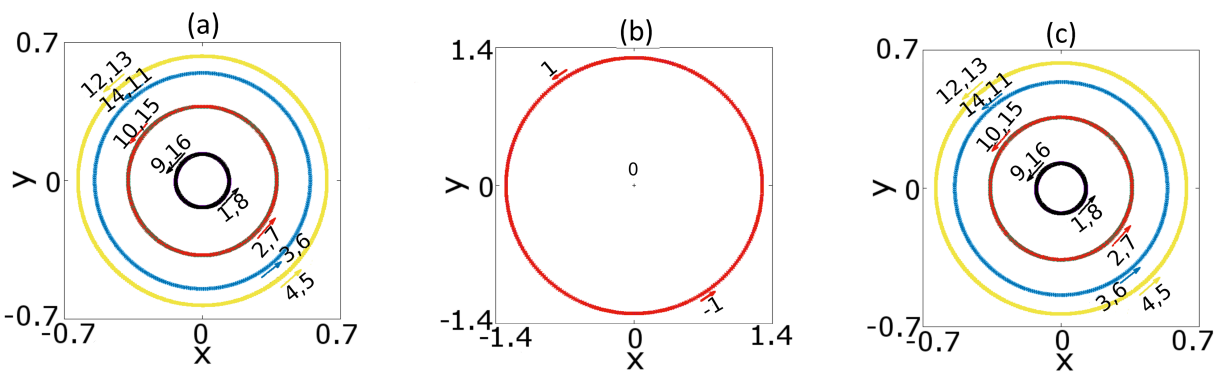

Fig. 7. Standing wave trajectories for $10 T \leqslant t \leqslant 13 T$ in the phase space $(x, y)$ of: (a) original system of $N=16$; (b) reduced system of $m=3$; (c) reduced system lifted to $N=16$. Other parameters: $\sigma=9, P=1, \lambda=1, \omega=2$.

linear part of the dynamic equations. From the dynamics in this reduced system, we are able to predict the dynamics lifted to the full system.

We have found generalized cluster synchronization patterns in the form of unstable standing waves, and asymptotically stable completely in-phase synchronized states.

\section{Acknowledgement}

This work was supported by DFG in the framework of SFB 910.

\section{Authors contribution statement}

LT did the numerical simulations, EP and LT did the theoretical analysis. EP contributed the Appendix. EP, AZ and ES designed and supervised the study. All authors contributed to the preparation of the manuscript. All the authors have read and approved the final manuscript.

\section{Appendix: Lyapunov function for the synchronization error}

In this Appendix we show that asymptotically the synchronization errors e converge to zero.

Consider a Lyapunov function given by

$$
V(\mathbf{e})=\frac{1}{2} \mathbf{e}^{*} \mathbf{e}
$$

where we recall that $\mathbf{e}=P \mathbf{z}, P^{*}=P=I_{N}-\mathbf{V}_{+} \mathbf{V}_{+}^{*}$, while the dynamics of $\mathbf{z}$ is given by (5):

$$
\mathbf{z}=-\mathbf{Z}^{(2)} \mathbf{z}+\frac{\sigma}{2 P} \tilde{L} \mathbf{z}=F^{n l}(\mathbf{z})+F^{(l)}(\mathbf{z})
$$

where we have split the dynamics into linear and nonlinear parts to simplify the proof, i.e., $F^{n l}(\mathbf{z})=-\mathbf{Z}^{(2)} \mathbf{z}$ and $F^{(l)}(\mathbf{z})=\frac{\sigma}{2 P} \tilde{L} \mathbf{z}$. terms

Using these notations the derivative of $V(\mathbf{e})$ can be presented as a sum of two

$$
\dot{V}(\mathbf{e})=\operatorname{grad} V(\mathbf{e})\left(F^{n l}(\mathbf{z})+F^{(l)}(\mathbf{z})\right) .
$$


First we consider the linear term for which, using the properties

$$
P=\mathbf{V}\left[\begin{array}{cc}
0 & 0 \\
0 & I_{N-3}
\end{array}\right] \mathbf{V}^{*}, \quad \mathbf{V}_{-}^{*} \mathbf{V}_{-}=I_{N-3}, \quad \mathbf{V}_{-} \mathbf{V}_{-}^{*}=I_{N}-\mathbf{V}_{+} \mathbf{V}_{+}^{*}
$$

we can write

$$
\begin{aligned}
\dot{V}^{(l)}(\mathbf{e}) & =\frac{\sigma}{2 P} \mathbf{e}^{*} P \tilde{L} \mathbf{z}=\frac{\sigma}{2 P} \mathbf{e}^{*} \mathbf{V}\left[\begin{array}{cc}
0 & 0 \\
0 & I_{N-3}
\end{array}\right] \mathbf{V}^{*} \mathbf{V}\left[\begin{array}{cc}
\Lambda_{+} & 0 \\
0 & \Lambda_{-}
\end{array}\right] \mathbf{V}^{*} \mathbf{z} \\
& =\frac{\sigma}{2 P} \mathbf{e}^{*} \mathbf{V}\left[\begin{array}{cc}
0 & 0 \\
0 & \Lambda_{-}
\end{array}\right] \mathbf{V}^{*} \mathbf{z}=\frac{\sigma}{2 P} \mathbf{e}^{*} \mathbf{V}_{-} \Lambda_{-} \mathbf{V}_{-}^{*} \mathbf{z}=\frac{\sigma}{2 P} \mathbf{e}^{*} \mathbf{V}_{-} \Lambda_{-} \mathbf{V}_{-}^{*} \mathbf{e}
\end{aligned}
$$

where in the last equality we have used $\mathbf{V}_{-}^{*} \mathbf{e}=\mathbf{V}_{-}^{*} \mathbf{z}$.

Recalling that all the eigenvalues of the matrix $\Lambda_{-}$have negative real parts, we obtain from (A.1)

$$
\dot{V}^{(l)}(\mathbf{e}) \leq-\gamma\|\mathbf{e}\|^{2},
$$

where $\gamma=\frac{\sigma}{2 P} \lambda_{\max }\left(\Lambda_{-}+\Lambda_{-}^{*}\right)$.

Next we consider the Lyapunov derivative for the nonlinear part. To start with, we use the definition of the projection matrix $P$ and the equality $P^{*} P=P$ to rewrite the Lyapunov function as

$$
V(\mathbf{e})=\frac{1}{2} \mathbf{e}^{*} \mathbf{e}=\frac{1}{2} \mathbf{z}^{*} P P^{*} \mathbf{z}=\frac{1}{2} \mathbf{z} P \mathbf{z}=\frac{1}{2} \mathbf{z}^{*}\left(I_{N}-\mathbf{V}_{+} \mathbf{V}_{+}^{*}\right) \mathbf{z} .
$$

Recalling that matrix $\mathbf{V}_{+}$is composed of the eigenvectors corresponding to the unstable directions, i.e. $\mathbf{V}_{+}=\left[\mathbf{1}, \mathbf{v}_{1}, \mathbf{v}_{-1}\right]$, where $\mathbf{1}^{\top}=[1, \ldots, 1]$, and $\mathbf{V}_{+} \mathbf{V}_{+}^{*}=$ $\mathbf{1 1} \mathbf{1}^{\top}+\mathbf{v}_{1} \mathbf{v}_{1}^{*}+\mathbf{v}_{-1} \mathbf{v}_{-1}^{*}$.

Using these expressions we can rewrite the function $V(\mathbf{e})$ as

$$
\begin{aligned}
V(\mathbf{e}) & =\frac{1}{2} \mathbf{z}^{*}\left(\mathbb{I}_{\mathbf{N}}-\mathbf{1} \mathbf{1}^{\top}\right) \mathbf{z}-\frac{1}{2}\left(\mathbf{z}^{*} \mathbf{v}_{1} \mathbf{v}_{1}^{*} \mathbf{z}+\mathbf{z}^{*} \mathbf{v}_{-1} \mathbf{v}_{-1}^{*} \mathbf{z}\right) \\
& =\frac{1}{2 N} \sum_{i, j=-N / 2}^{N / 2-1}\left(\overline{\mathbf{z}}_{i}-\overline{\mathbf{z}}_{j}\right)\left(\mathbf{z}_{i}-\mathbf{z}_{j}\right)-\frac{1}{2}\left(\mathbf{z}^{*} \mathbf{v}_{1} \mathbf{v}_{1}^{*} \mathbf{z}+\mathbf{z}^{*} \mathbf{v}_{-1} \mathbf{v}_{-1}^{*} \mathbf{z}\right) .
\end{aligned}
$$

We consider now in detail the last two terms above. Since by assumption $N$ is even, we can introduce a vector

$$
\xi^{\top}=\left[1, \exp \left(\frac{\pi i}{N}\right), \ldots, \exp \left(\frac{\pi i(N-1)}{N}\right)\right] \in \mathbb{R}^{N / 2}
$$

and in this case the eigenvectors $\mathbf{v}_{1}$ and $\mathbf{v}_{-1}$ have a very particular structure, notably

$$
\mathbf{v}_{1}=\frac{1}{\sqrt{N}}\left[\begin{array}{r}
\boldsymbol{\xi} \\
-\boldsymbol{\xi}
\end{array}\right] \quad \text { and } \quad \mathbf{v}_{-1}=\frac{1}{\sqrt{N}}\left[\begin{array}{r}
\boldsymbol{\xi}^{* T} \\
-\boldsymbol{\xi}^{* T}
\end{array}\right]
$$

In the same way we can split the vector $\mathbf{z}$ in two parts as $\mathbf{z}_{1}=\left(\mathbf{z}_{0}, \ldots, \mathbf{z}_{\frac{N}{2}-1}\right)$ and $\mathbf{z}_{2}=\left(\mathbf{z}_{-\frac{N}{2}}, \mathbf{z}_{-1}, \ldots, \mathbf{z}_{-\frac{N}{2}+1}\right)$. Using this new notation we can write the expression $\mathbf{z}^{*} \mathbf{v}_{1} \mathbf{v}_{1}^{*} \mathbf{z}$ in terms of $\boldsymbol{\xi}$ and $\mathbf{z}_{1}, \mathbf{z}_{2}$ as

$$
\mathbf{z}^{*} \mathbf{v}_{1} \mathbf{v}_{1}^{*} \mathbf{z}=\frac{1}{N}\left[\mathbf{z}_{1}^{*}, \mathbf{z}_{2}^{*}\right]\left[\begin{array}{cc}
\boldsymbol{\xi} \boldsymbol{\xi}^{*} & -\boldsymbol{\xi} \boldsymbol{\xi}^{*} \\
-\boldsymbol{\xi} \boldsymbol{\xi}^{*} & \boldsymbol{\xi} \boldsymbol{\xi}^{*}
\end{array}\right]\left[\begin{array}{c}
\mathbf{z}_{1} \\
-\mathbf{z}_{2}
\end{array}\right]
$$


Straightforward calculations show that

$$
\begin{aligned}
\mathbf{z}^{*} \mathbf{v}_{1} \mathbf{v}_{1}^{*} \mathbf{z} & =\left(\mathbf{z}_{1}-\mathbf{z}_{2}\right)^{*} \boldsymbol{\xi} \boldsymbol{\xi}^{*}\left(\mathbf{z}_{1}-\mathbf{z}_{2}\right)=\sum_{i=0}^{\frac{N}{2}-1} \mathbf{v}_{i} \mathbf{v}_{i}^{*}\left(\mathbf{z}_{i}^{*}-\mathbf{z}_{-i+1}\right)^{*}\left(\mathbf{z}_{i}^{*}-\mathbf{z}_{-i-1}\right) \\
& =\sum_{i=0}^{\frac{N}{2}-1} \mathbf{v}_{i} \mathbf{v}_{i}^{*}\left\|\mathbf{z}_{i}^{*}-\mathbf{z}_{-i+1}\right\|^{2}=\frac{1}{2} \sum_{i=0}^{\frac{N}{2}-1} \mathbf{v}_{i} \mathbf{v}_{i}^{*}\left\|\mathbf{z}_{i}^{*}-\mathbf{z}_{-i+1}\right\|^{2} \\
& +\frac{1}{2} \sum_{i=-\frac{N}{2}}^{-1} \mathbf{v}_{i} \mathbf{v}_{i}\left\|\mathbf{z}_{i}^{*}-\mathbf{z}_{-i-1}\right\|^{2}
\end{aligned}
$$

Repeating the same procedure for the term $\mathbf{z}^{*} \mathbf{v}_{-1} \mathbf{v}_{-1}^{*} \mathbf{z}$ we obtain

$$
\mathbf{z}^{*} \mathbf{v}_{-1} \mathbf{v}_{-1}^{*} \mathbf{z}=\frac{1}{2} \sum_{i=0}^{\frac{N}{2}-1} \mathbf{v}_{i}^{*} \mathbf{v}_{i}\left\|\mathbf{z}_{i}^{*}-\mathbf{z}_{-i+1}\right\|^{2}+\frac{1}{2} \sum_{i=-\frac{N}{2}}^{-1} \mathbf{v}_{i}^{*} \mathbf{v}_{i}\left\|\mathbf{z}_{i}^{*}-\mathbf{z}_{-i-1}\right\|^{2}
$$

Substituting the obtained expressions in Eq. (A.3) we find for the Lyapunov function

$$
\begin{aligned}
V(\mathbf{e}) & =\frac{1}{2 N} \sum_{i, j=-\frac{N}{2}}^{\frac{N}{2}-1}\left\|\mathbf{z}_{i}-\mathbf{z}_{j}\right\|^{2}-\frac{1}{4} \sum_{i=0}^{\frac{N}{2}-1}\left(\mathbf{v}_{i}^{*} \mathbf{v}_{i}+\mathbf{v}_{i} \mathbf{v}_{i}^{*}\right)\left\|\mathbf{z}_{i}-\mathbf{z}_{-i+1}\right\|^{2} \\
& -\frac{1}{4} \sum_{i=-\frac{N}{2}}^{-1}\left(\mathbf{v}_{i}^{*} \mathbf{v}_{i}+\mathbf{v}_{i} \mathbf{v}_{i}^{*}\right)\left\|\mathbf{z}_{i}-\mathbf{z}_{-i-1}\right\|^{2}=\frac{1}{2 N} \sum_{i, j=-\frac{N}{2}}^{\frac{N}{2}-1} a_{i j}\left\|\mathbf{z}_{i}-\mathbf{z}_{j}\right\|^{2}
\end{aligned}
$$

where

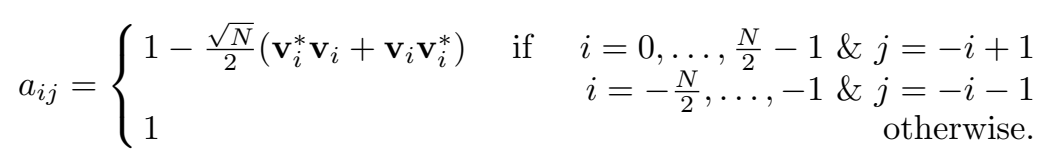

It is important to underline here that all coefficients $a_{i j} \geq 0$, hence the function $V(\mathbf{e})$ represents a sum of $\left\|\mathbf{z}_{i}-\mathbf{z}_{j}\right\|^{2}$ with nonnegative coefficients, i.e.,

$$
V(\mathbf{e})=\frac{1}{2 N} \sum_{i, j=-\frac{N}{2}}^{\frac{N}{2}-1} a_{i j}\left\|\mathbf{z}_{i}-\mathbf{z}_{j}\right\|^{2}
$$

Taking the derivative of this Lyapunov function for the nonlinear part of the system dynamics we obtain

$$
\dot{V}^{(n l)}(\mathbf{e})=\sum_{i, j} a_{i j}\left(\mathbf{z}_{i}-\mathbf{z}_{j}\right)^{*}\left(-\left\|\mathbf{z}_{i}\right\|^{2} \mathbf{z}_{i}+\left\|\mathbf{z}_{j}\right\|^{2} \mathbf{z}_{j}\right) .
$$

Each term in this sum can be estimated by bounds in the following way

$$
\begin{array}{r}
\left(\mathbf{z}_{i}-\mathbf{z}_{j}\right)^{*}\left(-\left\|\mathbf{z}_{i}\right\|^{2} \mathbf{z}_{i}+\left\|\mathbf{z}_{j}\right\|^{2} \mathbf{z}_{j}\right)=-\left\|\mathbf{z}_{i}\right\|^{4}-\left\|\mathbf{z}_{j}\right\|^{4}+\mathbf{z}_{i}^{*} \mid \mathbf{z}_{j}\left\|^{2} \mathbf{z}_{j}+\mathbf{z}_{j}^{*}\right\| \mathbf{z}_{i} \|^{2} \mathbf{z}_{i} \\
\leq-\left\|\mathbf{z}_{i}\right\|^{4}-\left\|\mathbf{z}_{j}\right\|^{4}+\frac{1}{2}\left\|\mathbf{z}_{i}\right\|^{4}+\left\|\mathbf{z}_{i}\right\|^{2}\left\|\mathbf{z}_{j}\right\|^{2}+\frac{1}{2}\left\|\mathbf{z}_{j}\right\|^{4} \leq 0
\end{array}
$$


where in the second line we used the bound

$$
\left\|\mathbf{z}_{j}\right\|^{2} \mathbf{z}_{i}^{*} \mathbf{z}_{j} \leq \frac{1}{2}\left\|\mathbf{z}_{j}\right\|^{4}+\frac{1}{2}\left\|\mathbf{z}_{i}^{*} \mathbf{z}_{j}\right\|^{2} \leq \frac{1}{2}\left\|\mathbf{z}_{j}\right\|^{4}+\frac{1}{2}\left\|\mathbf{z}_{i}\right\|^{2}\left\|\mathbf{z}_{j}\right\|^{2} .
$$

Finally, combining the derivatives for the linear and nonlinear parts given in Eqs. (A.1) and (A.6), we obtain

$$
\dot{V}(\mathbf{e})=\dot{V}^{(l)}(\mathbf{e})+\dot{V}^{(n l)}(\mathbf{e}) \leq-\gamma\|\mathbf{e}\|^{2}
$$

and therefore the synchronization errors e asymptotically tend to zero.

\section{References}

1. E. Panteley, A. Loria, IEEE Trans. Autom. Control, 62 (2017), https://doi: 10.1109/TAC.2017.2649382

2. J. van der Mark, B. van der Pol, Physica 1, 437 (1934), https://doi.org/10.1016/S00318914(34)90051-3

3. I.I. Blekhman, A.L. Fradkov, H. Nijmeijer, A.Y. Pogromsky, Syst. Control Lett. 31 (1997)

4. A. Pikovsky, M.G. Rosenblum, J. Kurths, Synchronization, A Universal Concept in Nonlinear Sciences (Cambridge University Press, Cambridge, 2001)

5. E. Mosekilde, Y. Maistrenko, D. Postnov, Chaotic Synchronization: Applications to Living Systems (World Scientific, Singapore, 2002)

6. A.G. Balanov, N.B. Janson, D.E. Postnov, O.V. Sosnovtseva, Synchronization: From Simple to Complex (Springer, Berlin, 2009)

7. T. Dahms, J. Lehnert, E. Schöll, Phys. Rev. E 86, 016202 (2012)

8. E. Schöll, in Advances in Analysis and Control of Time-Delayed Dynamical Systems (World Scientific, Singapore, 2013), Ed. by J.-Q. Sun, Q. Ding, chap. 4, pp. 57-83

9. V.I. Nekorkin, Introduction to Nonlinear Oscillations (Wiley, Weinheim, 2015)

10. E. Schöll, S.H.L. Klapp, P. Hövel, Control of self-organizing nonlinear systems (Springer, Berlin, 2016)

11. S. Boccaletti, A.N. Pisarchik, C.I. del Genio, A. Amann, Synchronization: From Coupled Systems to Complex Networks (Cambridge University Press, 2018), ISBN 9781107056268

12. E. Panteley, A. Loria, Proc. 6th IFAC Workshop on Periodic Control Systems (PSYCHO 2016), IFAC-PapersOnLine 49, 90 (2016), https://doi.org/10.1016/j.ifacol.2016.07.990

13. E. Panteley, A. Loria, A. El-Ati, Int. Journal of Control (2019) https://doi.org/10.1080/00207179.2018.1551618

14. Y. Kuramoto, D. Battogtokh, Nonlin. Phen. in Complex Sys. 5, 380 (2002)

15. F.M. Atay, Phys. Rev. Lett. 91, 094101 (2003)

16. B. Fiedler, V. Flunkert, P. Hövel, E. Schöll, Phil. Trans. R. Soc. A 368, 319 (2010)

17. C.U. Choe, T. Dahms, P. Hövel, E. Schöll, Phys. Rev. E 81, 025205(R) (2010)

18. Y.N. Kyrychko, K.B. Blyuss, E. Schöll, Phil. Trans. R. Soc. A 371, 20120466 (2013)

19. C.M. Postlethwaite, G. Brown, M. Silber, Phil. Trans. R. Soc. A 371, 20120467 (2013)

20. I. Schneider, Phil. Trans. R. Soc. A 371, 20120472 (2013)

21. L. Schmidt, K. Schönleber, K. Krischer, V. Garcia-Morales, Chaos 24, 013102 (2014)

22. A. Zakharova, M. Kapeller, E. Schöll, Phys. Rev. Lett. 112, 154101 (2014)

23. A. Zakharova, S. Loos, J. Siebert, A. Gjurchinovski, J.C. Claussen, E. Schöll, in Control Self Organ. Nonlinear Syst., edited by E. Schöll, S.H.L. Klapp, P. Hövel (Springer, Berlin, Heidelberg, 2016)

24. L. Illing, Phys. Rev. E 94, 022215 (2016)

25. A. Koseska, E. Volkov, J. Kurths, Phys. Rep. 531, 173 (2013)

26. A. Zakharova, I. Schneider, Y.N. Kyrychko, K.B. Blyuss, A. Koseska, B. Fiedler, E. Schöll, Europhys. Lett. 104, 50004 (2013) 
27. I. Schneider, M. Kapeller, S. Loos, A. Zakharova, B. Fiedler, E. Schöll, Phys. Rev. E 92, 052915 (2015)

28. A.Y. Pogromsky, T. Glad, H. Nijmeijer, Int. J. Bifurc. Chaos 9, 629 (1999)

29. A.Y. Pogromsky, H. Nijmeijer, IEEE Trans. Circuits Syst. I - Fundam. Theory Appl. 48, 152 (2001)

30. C. Wille, J. Lehnert, E. Schöll, Phys. Rev. E 90, 032908 (2014) 